\title{
Health human resources for emergency medicine: a framework for the future
}

\author{
Douglas Sinclair, MD, CCFP-EM, FRCPC ${ }^{* \dagger \neq}$; Peter Toth, MD, CCFP-EM ${ }^{\S}$; Alecs Chochinov, MD FRCPC ; \\ John Foote, MD, CCFP-EM, FCFP§®\#; Kirsten Johnson, MD, MPH, CCFP-EM\|; Jill McEwen, MD, \\ FRCPC $^{* *}$; David Messenger, MD, FRCPC ${ }^{\dagger+}$; Judy Morris, MD, FRCPC $\neq \neq$; Paul Pageau, MD, \\ CCFP-EM ${ }^{\S \S ;}$; David Petrie, MD, FRCPC $\pi$; Carolyn Snider, MD, MPH, FRCPC ${ }^{*}$
}

\section{ABSTRACT}

In June of 2016, the Collaborative Working Group (CWG) on the Future of Emergency Medicine presented its final report at the Canadian Association of Emergency Physicians (CAEP) annual meeting in Quebec City. The CWG report made a number of recommendations concerning physician Human Health Resource (HHR) shortfalls in emergency medicine, specific changes for both the Royal College of Physicians and Surgeons of Canada (FRCPC) and the College of Family Physicians of Canada (CCFP-EM) training programs, HHR needs in rural and remote hospitals, future collaboration of the CCFPEM and FRCPC programs, and directions for future research. All recommendations were endorsed by CAEP, the Royal College of Physicians and Surgeons of Canada (RCPSC), and the College of Family Physicians of Canada (CFPC). The CWG report was published in CJEM and has served as a basis for ongoing discussion in the emergency medicine community in Canada. The CWG identified an estimated shortfall of 478 emergency physicians in Canada in 2016, rising to 1071 by 2020 and 1518 by 2025 assuming no expansion of EM residency training capacity. In 2017, the CAEP board struck a new committee, The Future of Emergency Medicine in Canada (FEMC), to advocate with appropriate stakeholders to implement the CWG recommendations and to continue with this important work. FEMC led a workshop at CAEP 2018 in Calgary to develop a regional approach to HHR advocacy, recognizing different realities in each province and region. There was wide representation at this workshop and a rich and passionate discussion among those present. This paper represents the output of the workshop and will guide subsequent deliberations by FEMC. FEMC has set the following three goals as we work toward the overarching purpose to improve timely access to high quality emergency care: (1) to define and describe categories of emergency departments (EDs) in Canada, (2) define the full time equivalents required by category of ED in Canada, and (3) recommend the ideal combination of training and certification for emergency physicians in Canada. A fourth goal supports the other three goals: (4) urge further consideration and implementation of the CWG-EM recommendations related to coordination and optimization of the current two training programs. We believe that goals 1 and 2 can largely be accomplished by the CAEP annual meeting in 2020, and goal 3 by the CAEP annual meeting in 2021. Goal 4 is ongoing with both the RCPSC and the CFPC. We urge the EM community across Canada to engage with our committee to support improved access and EM care for all Canadians.

Key words: Human health resources, emergency medicine training

\section{INTRODUCTION}

In June of 2016, the Collaborative Working Group (CWG) on the Future of Emergency Medicine presented its final report at the Canadian Association of Emergency Physicians (CAEP) annual meeting in Quebec City. The CWG report made several recommendations concerning physician Human Health Resource

From the *St. Michael's Hospital, Toronto, ON, Canada; †Nova Scotia Health Authority, Halifax, NS, Canada; łUniversity of Toronto, Department of Medicine, Division of Emergency Medicine, Toronto, ON, Canada; §University of Toronto, Department of Family and Community Medicine, Toronto, ON, Canada; UUniversity of Manitoba, Department of Emergency Medicine, Winnipeg, MB, Canada; \#Schwartz-Reisman Emergency Centre, Mount Sinai Hospital, Toronto, ON, Canada; ||McGill University, Department of Emergency Medicine, Montreal, QC, Canada; **University of British Columbia, Department of Emergency Medicine, Vancouver, BC, Canada; ††Queen's University, Department of Emergency Medicine, Kingston, ON, Canada; ¥¥Laval University, Department of Emergency Medicine, Quebec City, QC, Canada; §§University of Ottawa, Department of Emergency Medicine, Ottawa, ON, Canada; and the IITDalhousie University, Department of Emergency Medicine, Halifax, NS, Canada.

Correspondence to: Dr. Douglas Sinclair, Department of Emergency Medicine, 1796 Summer Street, Suite 355, Halifax, NS B3H 3A7, Canada; Email: emergency.medicine@dal.ca

(C) Canadian Association of Emergency Physicians
CJEM 2020;22(1):40-44

DOI 10.1017/cem.2019.446 
(HHR) shortfalls in emergency medicine (EM), specific changes for both the Royal College of Physicians and Surgeons of Canada (FRCPC) and the College of Family Physicians of Canada (CCFP-EM) training programs, HHR needs in rural and remote hospitals, future collaboration of the CCFP-EM and FRCPC programs, and directions for future research. All recommendations were endorsed by CAEP, the Royal College of Physicians and Surgeons of Canada (RCPSC), and the College of Family Physicians of Canada (CFPC). The CWG report was published in $C 7 E M^{1}$ and has served as a basis for ongoing discussion in the emergency medicine community in Canada.

The CWG identified an estimated shortfall of 478 emergency physicians in Canada in 2016, rising to 1,071 by 2020 and 1,518 by 2025 , assuming no expansion of EM residency training capacity (Figure 1).

Decisions affecting residency training positions are made at the provincial Ministry of Health $(\mathrm{MOH})$ level, in collaboration with their prospective postgraduate (PG) medical school deans. Each province has a different approach to this work, and the decision-making process is under increased scrutiny as health systems struggle to maintain and improve services. What has hitherto been an opaque and "organic" process, often serving individual program needs, based on flawed historical (billing-based) full time equivalent (FTE) data and methodology, is now being held more accountable by Hospital/Regional Health Authority leaders, whose mandate is to serve the health care needs of the public.

In 2017, the CAEP board struck a new committee, the Future of Emergency Medicine in Canada (FEMC), to advocate with appropriate stakeholders to implement the CWG recommendations and to continue with this important work. As part of this work, FEMC led a workshop at CAEP 2018 in Calgary to develop a regional approach to HHR advocacy, recognizing different realities in each province and region.

It is clear from this workshop and subsequent meetings that FEMC must focus its research and advocacy efforts toward achieving the following overarching goal: "to optimize timely access to high quality emergency care for the patients and populations that we serve." As a means to that end, the following three goals will be the focus of this committee: (1) defining the various categories of emergency department (ED) care in Canada, (2) defining the numbers of FTEs required for each category, and (3) making recommendations for physician training and certification to meet the HHR needs for now and the future.

\section{CURRENT STATUS OF EM RESIDENCY PROGRAMS IN}

\section{CANADA}

Residency training programs in EM were established in Canada in the early 1980s as a response to serious concerns about the quality of care being provided in many emergency departments (EDs), and the significant gap in any formal training programs at the time. Over the years, they have evolved and filled an important need in our Health Systems, are well regarded by educational leaders, and are highly sought after by medical students. In 2018, the Canadian Resident Matching Service (CARMS) reported that PG year 1 Royal College of Physicians and Surgeons of Canada (RCPSC) positions in EM were in the highest demand of all residency positions. $^{3}$

The number of government-funded CCFP-EM spots nationally varies from a low of 110 to 132 spots per year with 127 spots offered in the 2018 match. ${ }^{4}$ These positions are also very popular, and each year approximately one-third of applicants are not matched.

Residency programs require both university infrastructure support and sufficient capacity in training sites to expand their numbers. A recent survey of both the FRCPC-EM and CCFP-EM program directors indicated that, on average, all programs can expand by two to three positions per year, for an approximate increase of 50 national EM certification positions, without new investments in infrastructure of expansion of training sites. Therefore, provincial $\mathrm{MOH}$ s could expand the number of EM training positions by re-allocating from other residency program positions, where there are a clear excess of limited employment opportunities or demand for training. Alternatively, MOHs could provide new investment to support EM. It must be noted that any expansion of the CCFP-EM program must include targeted EM funding for all 3 years of training, otherwise Canada faces the unintended consequence of the expansion of EM physicians with the reduction of graduation of new family physicians, who are vitally needed to support and advance primary care.

\section{UNIOUE CHALLENGES IN RURAL CANADA}

Staffing of EDs and other facilities that deliver emergency care in rural and remote areas of Canada is a difficult issue for MOHs and a serious concern for local communities. We know that a very small number of 


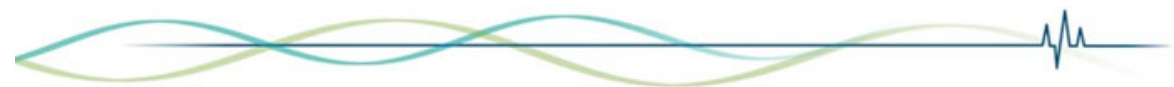

Estimate and future projections:

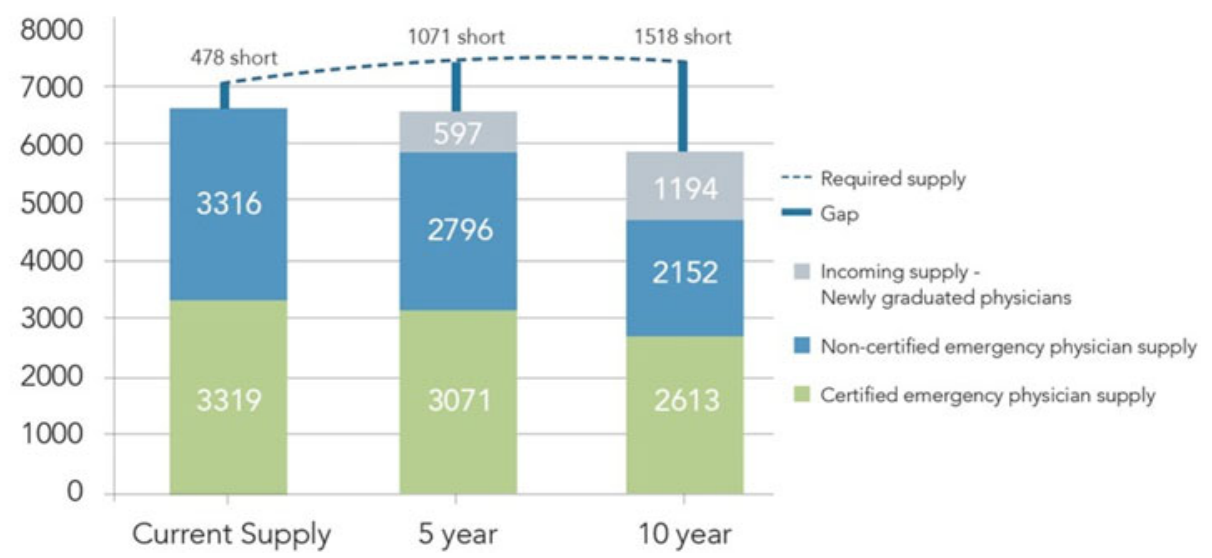

Figure 1. National emergency physician shortfall.

physicians with EM residency training practice in these resource-limited settings. Emergency care is typically provided by family physicians with EM competencies achieved in their 2-year family medicine (FM) residency, supplemented by related courses. The Society of Rural Physicians of Canada (SRPC), the CFPC, and CAEP have examined this issue both separately and in collaboration and suggest ways of developing novel approaches to both residency training and continuing professional development (CPD) to support physicians in the delivery of emergency care in these settings.

Apart from the necessary step of increasing training positions leading to EM certification, another focus must be to support the improvement of emergency care delivered by rural family physicians by developing mentorship and clinical experiences pathways that support achievement of practice-eligible EM certification.

It should be noted that these programs are in many ways an "interim solution" and have arisen due to the challenges faced by both the RCPS EM programs and the CCFP and CCFP-EM programs to address the needs of "patient zero" as referred to in the CWG report. The CWG group felt strongly that, at the end of residency training, "patient zero" should receive the same high standard of care by graduates of all three training programs.

Additional strategies include ongoing CPD support to rural physicians ranging from Advanced Trauma Life Support/Pediatric Advanced Life Support (ATLS/ PALS), to needs-based short programs using simulation (used by TREKK in the PedsPac program for pediatric $\mathrm{EM}),{ }^{5}$ and finally real-time support of clinical decision making through decision support networks such as the British Columbia Emergency Medicine Network and through provincial telemedicine links. ${ }^{6}$

\section{FRCPC v. CCFP-EM RESIDENCY TRAINING}

The CWG conducted an extensive survey of ED chiefs, ED physicians, and residents as part of their work. A substantial proportion of the survey respondents reported discontent with the current two residency training approaches and the narrative section displayed many passionate responses to this issue. Nevertheless, the CWG did not recommend a single training program. Both training programs attract high quality trainees and have strengths and limitations well outlined in the CWG report. The CFPC and the RCPSC see greater value in optimizing their individual programs at this time rather than the complex task of combining EM training programs into a single stream.

Both Colleges have accepted the CWG recommendations and have improved their collaboration with each other. The CFPC has committed to review the EM content and training in both the CCFP and CCFP-EM program and the RCPSC has currently implemented the Competency by Design program, which includes prescribed competencies that address the concerns raised by the CWG report. 
FEMC believes that there is an important role for graduates of each program and that continued dialogue on the value of one program over another is a distraction from the most important issue, that is, the ongoing shortage of residency training positions to support EM patients in the future. Working with the assumption that the two coordinated and optimized training programs would complement each other, and that the Emergency Health ecosystem is more robust and resilient when the relative ratios are optimally balanced between the two pathways, then we can thoughtfully address the question: what is the optimal ratio for each training program in EM Physician Resource Planning recommendations?

\section{CATEGORIZATION OF EDs}

Canada has a vast geography and variable population densities, which has a significant impact on the mix and distribution of EDs in a region. Busy urban full service EDs have different HHR needs than small rural EDs. The ideal number and mix of the physician types will be relative to the categorization and distribution of EDs and other emergency care facilities (which may not be defined as an ED per se) in a given region.

FEMC proposes that there are roughly four "levels" of EDs to be considered in planning a regional/provincial system. They include: Level 1, large urban tertiary/ teaching; Level 2, busy regional and/or suburban; Level 3, full service community; and Level 4, smaller rural. There will be smaller facilities where emergency care is delivered that may not actually qualify for the term "emergency department." The important point for this context is that HHR must occur in close alignment with Clinical Service Plans (CSP) so that the overarching goal is designing Health Systems to optimize timely access to high quality emergency care for the patients and populations that we serve.

\section{CONCLUSIONS}

The work of FEMC is to fundamentally answer the question "How do our health systems optimize timely access to high quality emergency care for the patients and populations that we serve?" Because having an adequate supply of emergency physicians is essential to answering that question, CAEP must take a leadership role in creating a principled vision for HHR planning, while advising on the multiple complexities and pragmatics of working toward such a vision.

We need to remind the EM community, the government funding bodies (i.e., provincial MOHs), and the faculties of medicine PG deans that, in 2016, the CWG predicted an HHR shortfall of 1,000 FTE in EM by 2020 and an over 1,500 FTE shortfall by 2025 . These 2016 estimates are proving accurate when compared with the real-life, real-time FTE shortages seen currently around the country in EDs. Existing EM training positions must be urgently increased by two to three positions per site, until the system is stabilized. Graduates of both the FRCPC and CCFP-EM programs are needed to meet the increasing demand in acuity and complexity of patient care in Canada. Categorization of EDs to define the type and number of ED physicians required is essential to inform provincial Clinical Service Plans for Emergency Medicine. A clear definition of an FTE in EM, and how it may be modifiable in various clinical (and clinical/academic) settings, is required and currently under way by FEMC.

Innovative approaches are required in rural Canada, which should include: focused CPD, real-time decision support, and a long-term goal of creating pathways to certification/competency specific to the local practice setting.

Final decisions on residency program numbers and clinical service plans for EM are made at a provincial and regional level, and ongoing advocacy at both the PG medical education and MOH level are required. Local advocacy will be the key to success, and the development of detailed, regional HHR plans is a critical goal.

In summary, FEMC has set the following three goals as we work toward the overarching purpose to improve timely access to high quality emergency care: (1) define and describe categories of EDs in Canada, (2) define the FTEs required by category of ED in Canada, and (3) recommend ideal combination of training and certification for emergency physicians in Canada. A fourth goal supports the other three goals: (4) urge further consideration and implementation of the CWG-EM recommendations related to coordination and optimization of the current two training programs.

We believe that goals 1 and 2 can largely be accomplished by the CAEP annual meeting in 2020 and goal 3 by the CAEP annual meeting in 2021. Goal 4 is ongoing with both the RCPSC and the CFPC. We invite Colleges, PG deans, and health services planners 
across Canada to engage with CAEP's FEMC to support improved access and EM care for all Canadians.

Supplementary material: The supplementary material for this article can be found at https://doi.org/10.1017/cem.2019.446.

\section{REFERENCES}

1. Sinclair D, Abu-Laban RB, Toth P, et al. Emergency medicine training and practice in canada: celebrating the past \& evolving for the future. Can 7 Emerg Med 2017;19(S2):S1S8.

2. McEwen J, Borreman S, Caudle J, et al. Position statement on emergency medicine definitions from the Canadian Association of Emergency Physicians. Can 7 Emerg Med 2018;20 (4):501-6.
3. Royal College of Physician and Surgeons Canada (Internet). Ottawa: The College; c2019 (cited April 25, 2019). The Royal College National Specialty Societies Conference Series. Available at: http://www.royalcollege.ca/rcsite/health-policy/ policy-conferences/royal-college-national-specialty-societiesconference-series-e (accessed October 16, 2019).

4. CaRMS. 2018 Family Medicine/Emergency Medicine Match. Table 1: Positions available and applicants by school of residency. (cited April 25, 2019). https://www.carms.ca/ wp-content/uploads/2018/06/fmem1e_2018.pdf (accessed October 16, 2019).

5. Translating Emergency Knowledge for Kids (Internet). Winnipeg: Trekk; c2019 (cited April 25, 2019). Pediatric Packages (PedsPacs). Available at: https://trekk.ca/browse_compendium_ resources?compendium_id=32 (accessed October 16, 2019).

6. Abu-Laban RB, Drebit S, Lindstrom RR, et al. The British Columbia Emergency Medicine Network: a paradigm shift in a provincial system of emergency care. Cureus 2018;10(1):e2022. 